PA'THOLOGISCHE

ANATONIE DER HAUSSÄUGETHIERE. 



\title{
PATHOLOGISCHE ANATOMIE
}

\author{
DER HAUSSÄUGETHIERE.
}

VON

\section{CHR. JOS. FUCHS,}

PROFESSOH AN DER OROSSIFRZOQLICH BADISCHEN THIKRAIRNEISCHULE IN CAKLSRUHE.

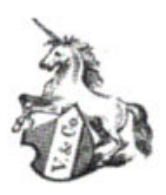

LEIPZIG, VERLAG VON VEIT \& COMP. 1859. 
